\title{
Novel hydrocarbonoclastic metal-tolerant Acinetobacter and Pseudomonas strains from Aconcagua river oil-polluted soil
}

\author{
Valentina Méndez ${ }^{1}$, Sebastián Fuentes ${ }^{1}$, Verónica Morgante ${ }^{1,2}$, Marcela Hernández ${ }^{1}$, \\ Myriam González ${ }^{1}$, Edward Moore ${ }^{3,4}$ and Michael Seeger ${ }^{1 *}$
}

${ }^{1}$ Laboratorio de Microbiología Molecular y Biotecnología Ambiental, Departamento de Química \& Centro de Biotecnología Daniel Alkalay Lowitt, Universidad Técnica Federico Santa María, Valparaíso, Chile. ${ }^{2}$ Programa Institucional de Fomento a la Investigación, Desarrollo e Innovación (PIDi), Universidad Tecnológica Metropolitana, Santiago, Chile. ${ }^{3}$ CCUG, Department of Clinical Bacteriology, Sahlgrenska University Hospital. ${ }^{4}$ Department of Infectious Diseases, The Sahlgrenska Academy of the University of Gothenburg, Gothenburg, Sweden.*Corresponding author: michael.seeger@usm.cl

\begin{abstract}
Bioremediation of sites polluted with petroleum hydrocarbons and heavy metals is a major challenge. The aim of this study was the isolation and characterization of hydrocarbon-degrading and heavy metal-tolerant bacteria. Sixteen hydrocarbonoclastic bacteria were isolated by enrichment from a crude oil-contaminated soil at Aconcagua river mouth, Central Chile. Most strains were cocci-shaped and exhibited circular cream-colored colonies with smooth texture. Isolates were resistant to bacitracin and penicillin, and two isolates were motile. Isolates were identified by $16 \mathrm{~S}$ rRNA and $r p o D$ and $r p o B$ genes sequence analyses. Most isolates belonged to Gammaproteobacteria including Acinetobacter radioresistens (4 isolates), Acinetobacter calcoaceticus (1), Pseudomonas stutzeri (2) and Pseudomonas chloritidismutans (1). Seven isolates possessed 97\% 16S rRNA gene similarity with A. calcoaceticus ATCC 23055T, suggesting that these probably represent a new Acinetobacter species. One isolate is an Actinobacteria of the Kocuria genus. All isolates were able to grow on crude oil, whereas eleven Acinetobacter and Pseudomonas strains grew on n-hexadecane. Pseudomonas isolates grew on fluorene (DM88 and DM95) and naphthalene (DD74). Acinetobacter isolates grew on fluorene (DD75, DD79 and DM81) and phenanthrene (DM82). Remarkably, most isolates (except DD79) exhibited copper or cadmium tolerance. These novel hydrocarbonoclastic and heavy metal-tolerant Pseudomonas and Acinetobacter strains are potential biocatalysts for bioremediation.
\end{abstract}

Keywords: Biodegradation, Acinetobacter, Pseudomonas, hydrocarbon, heavy metal, PAH 


\section{Introduction}

Petroleum hydrocarbons are main pollutants in the environment. A complex combination of aliphatic, (ali)cyclic and polyaromatic hydrocarbons (PAHs) are present in petroleum hydrocarbon mixtures such as crude oil, motor oil and diesel fuel (Fuentes et al., 2014). Heavy metals such as copper, cadmium, mercury, lead and nickel are co-pollutants frequently present in hydrocarbon-contaminated sites (Amezcua-Allieri et al., 2005; Olaniran et al., 2013; Fuentes et al., 2015). Mining, agriculture and industrial activities, contribute with persistent organic pollutants (POPs) and heavy metal release into the environment influencing the microbial communities (Hernández et al., 2011; Altimira et al., 2012; Fuentes et al., 2014; 2015). The Aconcagua River mouth in central Chile has been affected by crude oil pollution mainly associated to a petroleum refinery located next to the seashore. In 2002, high amounts of crude oil (70,000 L) were accidentally discharged into Aconcagua river mouth. High levels of PHAs were detected in the coastal reef next to the Aconcagua river mouth (Palma-Fleming et al., 2008). Bioremediation is an attractive technology for removal of POPs from the environment (Atlas and Philp, 2005; Morgante et al., 2010; Seeger et al., 2010; Ponce et al., 2011). Degradation of alkanes by Alcanivorax borkhumensis SK2, Pseudomonas putida GPo1 and Acinetobacter baylyi ADP1 has been characterized (Ratajczak et al. 1998; van Beilen et al., 2001). P. putida strains mt-2 and G7, and Acidovorax sp. NA3 are capable of degrading aromatic hydrocarbons (Velázquez et al., 2006; Singleton et al., 2009). Bioremediation using hydrocarbon-degrading strains has been successfully applied for crude-oil removal from environments (Silva et al., 2009; Fuentes et al., 2014; 2016). However, co-contaminated environments with heavy metals and organic pollutants are major challenges for bioremediation purposes. It has been reported that heavy metals inhibit microbial catabolic enzymes (Olaniran et al., 2013). Therefore, the isolation and characterization of heavy-metal tolerant bacteria for bioremediation of environments polluted with petroleum hydrocarbons and heavy metals is crucial. For the identification of bacterial isolates and to determine their phylogenetic relationships, genotypic characterization based on PCR amplification and sequence analyses of 16S rRNA genes has been commonly used. However, 16S rRNA gene sequence analyses often do not provide the resolution for definitive species-level identifications. Analyses of house keeping $r p o B$ and $r p o D$ gene sequences is useful for a more exhaustive bacterial identification (Moore et al., 2010).

Thus, the aim of this study was the isolation, identification and characterization of hydrocarbonoclastic and heavy metal-tolerant bacterial strains from a crude oil-contaminated soil at the Aconcagua river mouth in Central Chile.

\section{Materials and Methods}

\subsection{Chemicals}

Aromatic and aliphatic hydrocarbons were purchased from Merck (Darmstadt, Germany). $\mathrm{HgCl}_{2}$, $\mathrm{CuCl}_{2}, \mathrm{NiCl}_{2}, \mathrm{CdCl}_{2}, \mathrm{~Pb}\left(\mathrm{NO}_{3}\right)_{2}$ (analytical grade) were obtained from Sigma Aldrich (Saint Louis, MO, USA). Antibiotic test discs were acquired from Arlab (Santiago, Chile).

\subsection{Bacterial growth and media}

Bacterial strains were grown in Tryptic Soy Broth (TSB) or Bushnell-Haas Broth (BHB) minimal medium using hydrocarbons as sole carbon sources. BHB medium contained ( $\left.\mathrm{L}^{-1}\right): 1 \mathrm{~g} \mathrm{~K}_{2} \mathrm{HPO}_{4} ; 1 \mathrm{~g} \mathrm{KH}_{2} \mathrm{PO}_{4} ; 1 \mathrm{~g} \mathrm{NH}_{4} \mathrm{NO}_{3}, 0.05$ $\mathrm{g} \mathrm{FeCl}_{3}, 0.2 \mathrm{~g} \mathrm{MgSO}_{4} \cdot 7 \mathrm{H}_{2} \mathrm{O}, 0.02 \mathrm{~g} \mathrm{CaCl}_{2}$ (Fuentes et 
al., 2016). Bacterial growth on hydrocarbons was determined in 96-well microtiter plates using the respiration indicator, 2,3,5-triphenyl-2H-tetrazolium chloride (TTC) as described by Fuentes et al. (2016) with modifications. BHB medium was supplemented with $0.6 \%\left(\mathrm{v} \mathrm{v}^{-1}\right) n$ octane or $n$-hexadecane, $0.05 \%\left(\mathrm{w} \mathrm{v}^{-1}\right)$ naphthalene, fluorene, anthracene or phenanthrene, or cyclohexane and toluene in gas phase as sole carbon source. Bacterial growth on TSB and BHB media without carbon source were used as controls. After incubation for $10 \mathrm{~d}$ at $30^{\circ} \mathrm{C}$, TTC and glucose, succinate or pyruvate were added. For heavy metal tolerances, Tris-buffered mineral salts (LPTMS) medium was used. LPTMS medium contained $\left(\mathrm{L}^{-1}\right)$ : $6.06 \mathrm{~g}$ Tris, $4.68 \mathrm{~g} \mathrm{NaCl}, 1.49 \mathrm{~g} \mathrm{KCl}, 1.07 \mathrm{~g} \mathrm{NH}_{4} \mathrm{Cl}, 0.43$ $\mathrm{g} \mathrm{Na}_{2} \mathrm{SO}_{4}, 0.2 \mathrm{~g} \mathrm{MgCl}_{2} \cdot 6 \mathrm{H}_{2} \mathrm{O}, 0.03 \mathrm{~g} \mathrm{CaCl}_{2} \cdot 2 \mathrm{H}_{2} \mathrm{O}, 0.23 \mathrm{~g}$ $\mathrm{Na}_{2} \mathrm{HPO}_{4} \cdot 12 \mathrm{H}_{2} \mathrm{O}, 0.005 \mathrm{~g} \mathrm{Fe}(\mathrm{III})(\mathrm{NH} 4)$ citrate, and 1 $\mathrm{ml}$ of the trace element solution SL7 of Biebl and
Pfenning (Rojas et al., 2011).

\subsection{Enrichment and isolation of hydrocarbon-de- grading bacteria}

Hydrocarbonoclastic strains were isolated from a sampled contaminated soil by enrichment, using collected crude oil from a decantation sludge (DD) or a motor oil (DM) as sole carbon sources. Surface soil samples $(0-20 \mathrm{~cm})$ were collected from a crude oil-polluted site at the Aconcagua river mouth $\left(32^{\circ} 55^{\prime} 27^{\prime}\right.$ 'S, $\left.71^{\circ} 30^{\prime} 15^{\prime \prime} \mathrm{W}\right)$, Valparaíso region (Figure 1a) and stored at $4{ }^{\circ} \mathrm{C}$. In addition, to high levels of total petroleum hydrocarbons (TPH) in this area, high levels of copper $\left(147 \mathrm{mg} \mathrm{kg}^{-1}\right)$ and arsenic (34 $\mathrm{mg} \mathrm{kg}^{-1}$ ) have been reported (Fuentes et al., 2015). Enumeration of hydrocarbon-degrading a)

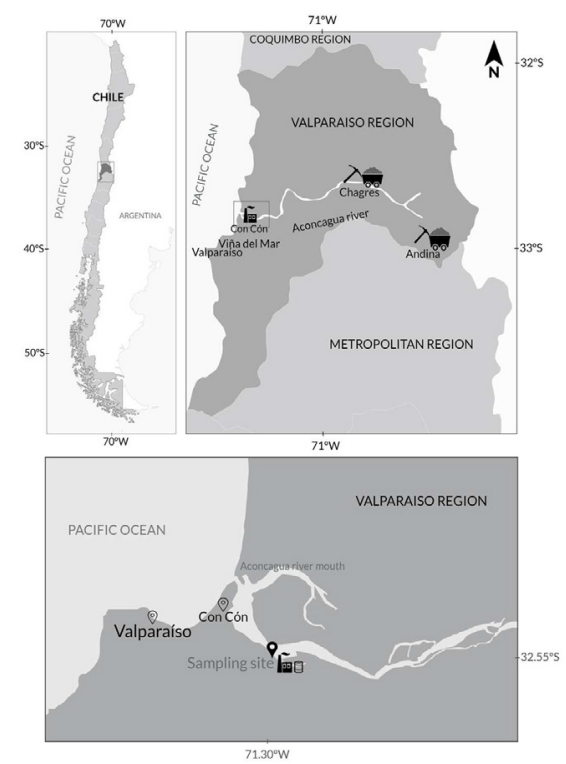

b)
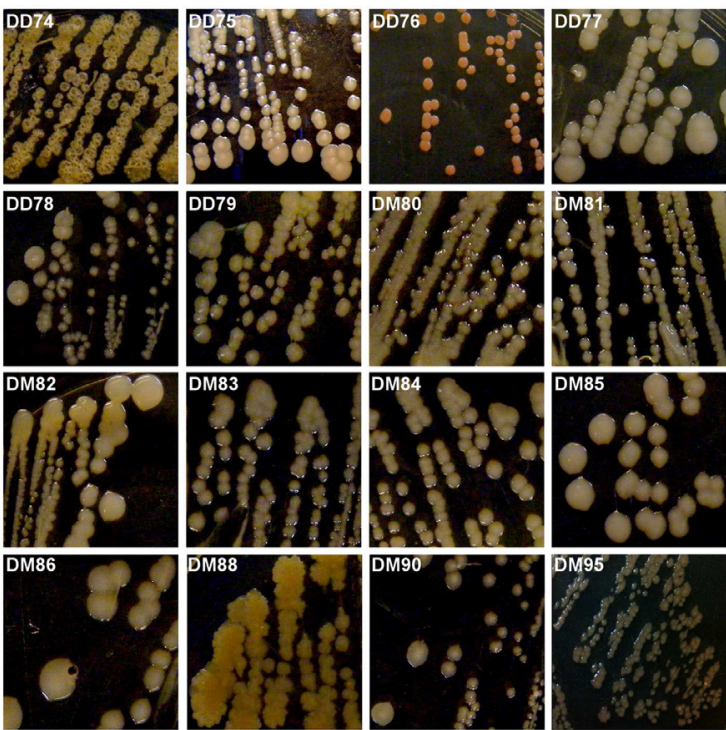

Figure 1. Isolation and characterization of hydrocarbonoclastic bacteria. (a) Sampling site at Aconcagua river in Valparaiso region, Central Chile is indicated with a black symbol. Industrial activities such as an oil refinery and copper mining industries are shown, (b) Colony morphology of hydrocarbonoclastic strains isolated, by enrichment using crude oil from decantation sludge (DD) from sampled soil, or motor oil (DM), as sole carbon and energy source are shown. 
microorganisms in soil was performed, using TTC indicator and the most-probable-number (MPN) enumeration in microtiter plates (Fuentes et al., 2016). with modifications. Briefly, $1 \mathrm{~g}$ of soil was suspended in BHB medium and agitated vigorously at room temperature for $2 \mathrm{~h}$. After decantation at $4^{\circ} \mathrm{C}$ for $2 \mathrm{~h}$, serial dilutions were prepared from supernatant aliquots, using BHB medium containing a solution of $2.5 \%$ crude oil in acetone $(1: 1)$. Microtiter plates were incubated with agitation at $30{ }^{\circ} \mathrm{C}$, and after $7 \mathrm{~d}, 0.01 \%$ TTC indicator was added. Ten grams of soil were added to 90 $\mathrm{mL}$ of BHB medium and $1 \%\left(\mathrm{v} \mathrm{v}^{-1}\right)$ oil (DD or DM) as sole carbon source. Prior to inoculation, DD and DM were sterilized at $180^{\circ} \mathrm{C}$ for $1 \mathrm{~h}$. Cycloheximide (150 $\left.\mathrm{mg} \mathrm{L}^{-1}\right)$ was added during enrichment to inhibit eukaryotic cell growth. Enrichments were incubated with agitation for $7 \mathrm{~d}$ at $30{ }^{\circ} \mathrm{C}$. Enrichment cultures were subcultured on fresh medium at 7-day intervals. Isolates were obtained after three subcultures by spreading onto $\mathrm{BH}$-agar plates with $1 \%\left(\mathrm{v} \mathrm{v}^{-1}\right)$ crude oil, and purified by streaking on TSA agar medium at $30^{\circ} \mathrm{C}$. Purity of strains was determined by colony morphology examination and by optical microscopy. Bacterial strains were named based on the crude oil used for enrichment (DD; crude oil from decantation sludge, DM; motor oil).

\subsection{Phenotypic and biochemical characterization}

Cells grown on TSA medium were observed by optical microscopy (Leica Galen III Microscope, USA). Motility and Gram-staining were determined. Briefly, M9 minimal medium containing $0.3 \%$ agar, was supplemented with $0.2 \%$ succinate, $0.1 \%$ yeast extract and $0.05 \%$ TTC indicator. Agar plates were inoculated and incubated overnight at $30^{\circ} \mathrm{C}$ in a humidified container. Catalase activity was determined by adding $30 \% \mathrm{H}_{2} \mathrm{O}_{2}$ in a slide containing a colony suspension in $0.9 \% \mathrm{NaCl}$ solution. Antibiotic resistance profiles were performed in Mueller Hinton agar medium (Difco, New Jersey, USA), using test discs containing bacitracin $(0.04 \mathrm{U})$, gentamicin $(10 \mu \mathrm{g})$, erythromycin $(15 \mu \mathrm{g})$, trimethoprim $(5 \mu \mathrm{g})$, streptomycin $(10 \mu \mathrm{g})$, kanamycin $(30 \mu \mathrm{g})$, penicillin (10 U), rifampicin (30 $\mu \mathrm{g})$, ampicillin $(10 \mu \mathrm{g})$ and tetracycline $(30 \mu \mathrm{g})$. Antibiotic resistance profiles were determined after incubation at $30^{\circ} \mathrm{C}$ for $12-14 \mathrm{~h}$.

\subsection{Tolerance to heavy metals}

Tolerance to heavy metals was determined in LPTMS agar medium ( $\mathrm{pH} 7.0$ ), supplemented with $0.2 \%$ sodium succinate and $0.1 \%$ yeast extract. The metal salts were added, in solution, at $0.53,0.9,1.2,1.4,1.6$ and $2.4 \mathrm{mmol} \mathrm{l}^{-1} \mathrm{Cu}^{+2} ; 0.85,1.0,1.3,1.5,1.7$ and 2.6 mmol l-1 $\mathrm{Ni}^{+2} ; 0.2,0.5,1.0,1.45$ and $2.4 \mathrm{mmol} \mathrm{l}^{-1} \mathrm{~Pb}^{+2}$; $0.22,0.53,0.9,1.3,1.8$ and $2.7 \mathrm{mmol} \mathrm{l}^{-1} \mathrm{Cd}^{+2}$ and 0.01 , 0.02 and $0.05 \mathrm{mmol} \mathrm{l}^{-1} \mathrm{Hg}^{+2}$ (Nies, 1999; Rojas et al., 2011). Heavy metal tolerances were determined by the presence or absence of growth after $5 \mathrm{~d}$ incubation at $30{ }^{\circ} \mathrm{C}$. Minimal inhibitory concentrations (MIC) were determined as described (Nies, 1999; Rojas et al., 2011) and plates were incubated at $28^{\circ} \mathrm{C}$ for $5 \mathrm{~d}$. The lowest concentration of metal salts that prevented growth was determined as the MIC (triplicate).

\subsection{Identification and phylogenetic analyses}

DNA was extracted by suspending a "loopfull" of the biomaterial in $100 \mu \mathrm{l}$ of TE buffer and incubated at $95^{\circ} \mathrm{C}$ for $10 \mathrm{~min}$. After centrifugation, supernatant was transfer to a clean tube and stored at $-20{ }^{\circ} \mathrm{C}$ for further analyses. The 16S rRNA genes were amplified by PCR using $27 \mathrm{f}$ (5'-AGAGTTTGATCMTGGCTCAG-3') and 1492r primers (5'- TACGGYTACCTTGTTACGACTT-3'). PCR conditions were performed as described previously (Altimira et al., 2012). PCR products were purified using Qiagen kit (Hilden, Germany), and sequenced 
at CCUG, University of Gothenburg (Gothenburg, Sweden) and Macrogen (Seoul, Korea). For Pseudomonas strains, partial sequencing of RNA polymerase subunit B (rpoB) (LAPS/LAPS27: 5'-TGGCCGAGAACCAGTTCCGCGT-3' and 5'-CGGCTTCGTCCAGCTTGTTCAG-3') and RNA polymerase subunit D (rpoD) (PsEG30F/PsEG790R: 5'-ATYGAAATCGCCAARCG-3' and 5'-CGGTTGATKTCCTTGA-3') was done. For Acinetobacter strains, $r p o B$ genes $(\mathrm{Ac} 1055 \mathrm{~F} /$ Ac1598R: 5'-GTGATAARATGGCBGGTCGT-3' and 5'-CGBGCRTGCATYTTGTCRT-3') were sequenced. 16S rRNA gene sequences were deposited at the European Bioinformatics Institute (EBI). The $r p o B$ and $r p o D$ gene sequences were deposited at NCBI. Phylogenetic trees were constructed based on comparative analyses of $16 \mathrm{~S}$ rRNA, $r p o B$ and $r p o D$ genes.

\section{Results}

\subsection{Isolation and phenotypic characterization}

In this study, sixteen hydrocarbon-degrading bacteria were isolated from a crude oil-polluted soil sampled close to the Aconcagua river mouth. This highly polluted soil contained $1 \times 10^{4}$ hydrocarbon-degrading bacteria per $g$ of dry soil. Hydrocarbonoclastic strains were isolated by enrichment after three subcultures with crude oil from a decantation sludge (DD) or a motor oil (DM). According to Gram-staining analyses, isolates were Gram-negative, except strain DD76. Most strains were cocci-shaped, whereas DD74, DM88 and DM95 were rod-shaped (Table 1).

Table 1. Phenotypic features and antibiotic resistance of hydrocarbonoclastic bacterial strains isolated close to the Aconcagua river mouth

\begin{tabular}{|c|c|c|c|c|c|c|c|c|c|c|c|c|c|c|c|c|}
\hline Strain & $\stackrel{+}{\hat{0}}$ & $\stackrel{n}{\hat{ิ}}$ & $\begin{array}{l}\stackrel{0}{\hat{\theta}} \\
\text { }\end{array}$ & $\hat{\hat{⿵}}$ & $\stackrel{\infty}{\stackrel{\circ}{\widehat{~}}}$ & $\begin{array}{l}\hat{\hat{\theta}} \\
\text { }\end{array}$ & 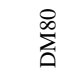 & 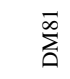 & $\sum_{0}^{\infty}$ & $\sum_{0}^{\infty}$ & $\sum_{a}^{+\infty}$ & $\sum_{0}^{\infty}$ & 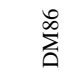 & $\sum_{0}^{\infty}$ & $\sum_{0}^{\varrho}$ & $\stackrel{a}{a}$ \\
\hline \multicolumn{17}{|c|}{ Phenotypic characteristics } \\
\hline Cell morphology & rod & cocci & cocci & cocci & cocci & cocci & cocci & cocci & cocci & cocci & cocci & cocci & cocci & rod & cocci & rod \\
\hline Gram stain & - & - & + & - & - & - & - & - & - & - & - & - & - & - & - & - \\
\hline Catalase & + & + & + & + & + & + & + & + & + & + & + & + & + & + & + & + \\
\hline Motility & + & - & + & - & - & - & - & - & - & - & - & - & - & - & - & - \\
\hline Colony shape ${ }^{*}$ & $\mathrm{i}$ & $\mathrm{c}$ & $\mathrm{c}$ & $\mathrm{c}$ & $\mathrm{c}$ & $\mathrm{c}$ & $\mathrm{c}$ & $\mathrm{c}$ & $\mathrm{c}$ & $\mathrm{c}$ & $\mathrm{c}$ & $\mathrm{c}$ & $\mathrm{c}$ & $\mathrm{i}$ & $\mathrm{c}$ & $\mathrm{c}$ \\
\hline Texture $^{*}$ & $\mathrm{r}$ & $\mathrm{s}$ & $\mathrm{s}$ & $\mathrm{s}$ & $\mathrm{s}$ & $\mathrm{s}$ & $\mathrm{s}$ & $\mathrm{s}$ & $\mathrm{s}$ & $\mathrm{s}$ & $\mathrm{s}$ & $\mathrm{s}$ & $\mathrm{s}$ & $\mathrm{r}$ & $\mathrm{s}$ & $\mathrm{r}$ \\
\hline Color ${ }^{*}$ & br & $\mathrm{cr}$ & rp & $\mathrm{cr}$ & $\mathrm{cr}$ & $\mathrm{cr}$ & $\mathrm{cr}$ & $\mathrm{cr}$ & $\mathrm{cr}$ & $\mathrm{cr}$ & $\mathrm{cr}$ & $\mathrm{cr}$ & $\mathrm{cr}$ & $\mathrm{pb}$ & $\mathrm{cr}$ & $\mathrm{pb}$ \\
\hline \multicolumn{17}{|c|}{ Antibiotic resistance $^{\dagger}$} \\
\hline Bacitracin & $\mathrm{R}$ & $\mathrm{R}$ & $\mathrm{R}$ & $\mathrm{R}$ & $\mathrm{R}$ & $\mathrm{R}$ & $\mathrm{R}$ & $\mathrm{R}$ & $\mathrm{R}$ & $\mathrm{R}$ & $\mathrm{R}$ & $\mathrm{R}$ & $\mathrm{R}$ & $\mathrm{R}$ & $\mathrm{R}$ & $\mathrm{R}$ \\
\hline Gentamicin & $S$ & $\mathrm{~S}$ & $\mathrm{~S}$ & $\mathrm{~S}$ & $\mathrm{~S}$ & S & S & $S$ & S & $\mathrm{S}$ & $\mathrm{S}$ & $\mathrm{S}$ & $S$ & $S$ & $\mathrm{~S}$ & $\mathrm{~S}$ \\
\hline Erythromycin & S & $\mathrm{R}$ & $S$ & S & S & S & $S$ & $S$ & $\mathrm{~S}$ & S & S & $\mathrm{S}$ & $S$ & $S$ & $S$ & $\mathrm{~S}$ \\
\hline Trimetropin & $S$ & $S$ & $S$ & $\mathrm{~S}$ & $\mathrm{~S}$ & $S$ & $S$ & $S$ & $\mathrm{~S}$ & $\mathrm{~S}$ & $\mathrm{~S}$ & $\mathrm{~S}$ & $S$ & $S$ & $S$ & $\mathrm{~S}$ \\
\hline Streptomycin & S & $\mathrm{S}$ & $\mathrm{S}$ & $\mathrm{S}$ & S & S & $\mathrm{S}$ & S & $\mathrm{S}$ & $\mathrm{S}$ & $\mathrm{S}$ & $\mathrm{S}$ & S & S & $\mathrm{S}$ & $\mathrm{S}$ \\
\hline Kanamycin & $S$ & $\mathrm{~S}$ & $\mathrm{R}$ & $\mathrm{S}$ & $S$ & S & $\mathrm{S}$ & S & S & $S$ & $\mathrm{~S}$ & $\mathrm{~S}$ & S & S & S & $\mathrm{S}$ \\
\hline Penicilin & $\mathrm{R}$ & $\mathrm{R}$ & $\mathrm{R}$ & $\mathrm{R}$ & $\mathrm{R}$ & $\mathrm{R}$ & $\mathrm{R}$ & $\mathrm{R}$ & $\mathrm{R}$ & $\mathrm{R}$ & $\mathrm{R}$ & $\mathrm{R}$ & $\mathrm{R}$ & $\mathrm{R}$ & $\mathrm{R}$ & $\mathrm{R}$ \\
\hline Rifampicin & S & S & S & $\mathrm{S}$ & $\mathrm{S}$ & S & S & S & $\mathrm{S}$ & $\mathrm{S}$ & $\mathrm{S}$ & $\mathrm{S}$ & $\mathrm{S}$ & S & S & $\mathrm{S}$ \\
\hline Ampicilin & S & S & $\mathrm{R}$ & $\mathrm{S}$ & $\mathrm{R}$ & $S$ & $\mathrm{R}$ & $\mathrm{R}$ & $\mathrm{R}$ & $S$ & $\mathrm{~S}$ & $\mathrm{~S}$ & $\mathrm{R}$ & $S$ & $\mathrm{R}$ & $\mathrm{S}$ \\
\hline Tetracycline & $\mathrm{S}$ & $\mathrm{R}$ & $\mathrm{R}$ & $\mathrm{R}$ & $\mathrm{R}$ & S & $\mathrm{R}$ & $\mathrm{R}$ & $\mathrm{R}$ & $\mathrm{R}$ & $\mathrm{S}$ & $\mathrm{R}$ & $\mathrm{R}$ & $\mathrm{S}$ & $\mathrm{R}$ & $\mathrm{S}$ \\
\hline
\end{tabular}

${ }^{*}$ Cells grown on TSA at $30^{\circ} \mathrm{C}$ for $24 \mathrm{~h}$. Colony shape: i, irregular; c, circular. Texture: r, rough; s, smooth. Color: br, brown; pb, pale-brown; cr, cream; rp, reddish-pink. $†$ S, sensitive; R, resistant. 
Most of the isolates exhibited cream-colored colonies with circular shape and smooth texture (Figure 1b). Only isolates DD74 and DM88 showed colonies with irregular borders (Figure 1b). Isolates DD74 and DM88 exhibited brown colonies, and strain DM95 showed pale-brown colonies, with rough and dry texture. Strain DD76 formed reddish-pink colonies. All isolates were catalase positive, and only DD74 and DD76 strains were motile. All isolates were resistant to bacitracin and penicillin. Additional resistance to kanamycin, ampicillin and tetracycline were observed in Gram-positive strain DD76, whereas Gram-negative strain DD75 possessed additional resistance to erythromycin and tetracyclin (Table 1).

Table 2. Identification of hydrocarbonoclastic bacterial strains isolated close to the Aconcagua river mouth by comparative sequence analyses.

\begin{tabular}{|c|c|c|c|}
\hline Isolate & $\begin{array}{l}\text { Accession } \\
\text { No. }^{\text {a,b }}\end{array}$ & Closest type strain (Accession No.) & Score (\% Identity) \\
\hline \multicolumn{4}{|c|}{ 16S rRNA gene ${ }^{a}$} \\
\hline DD74 & LN871431 & $\begin{array}{l}\text { Pseudomonas stutzeri ATCC17588 } \\
\text { (AF094748.1) }\end{array}$ & $1281 / 1281(100)$ \\
\hline DD75 & LN871432 & $\begin{array}{l}\text { Acinetobacter radioresistens DSM6976 } \\
\text { (X81666.1) }\end{array}$ & 460/465 (99) \\
\hline DD76 & LN871433 & Kocuria rosea DSM20447 (X87756.1) & $447 / 450(99)$ \\
\hline DD77 & LN871434 & A. calcoaceticus ATCC23055 (AJ888983.1) & $457 / 470(97)$ \\
\hline DD78 & LN871435 & A. radioresistens DSM6976 (X81666.1) & 460/465 (99) \\
\hline DD79 & LN871436 & A. radioresistens DSM6976 (X81666.1) & $1000 / 1002(99)$ \\
\hline DM80 & LN871437 & A. calcoaceticus ATCC23055 (AJ888983.1) & $461 / 473(97)$ \\
\hline DM81 & LN871438 & A. calcoaceticus ATCC23055 (AJ888983.1) & $461 / 473(97)$ \\
\hline DM82 & LN871439 & A. calcoaceticus ATCC23055 (AJ888983.1) & $458 / 470(97)$ \\
\hline DM83 & LN871440 & A. calcoaceticus ATCC23055 (AJ888983.1) & $460 / 472(97)$ \\
\hline DM84 & LN871441 & A. calcoaceticus ATCC23055 (AJ888983.1) & $1289 / 1301(99)$ \\
\hline DM85 & LN871442 & A. calcoaceticus ATCC23055 (AJ888983.1) & $458 / 473(97)$ \\
\hline DM86 & LN871443 & A. calcoaceticus ATCC23055 (AJ888983.1) & $458 / 470(97)$ \\
\hline DM88 & LN871445 & P. chloritidismutans AW-1 (NR_115115.1) & $466 / 469(99)$ \\
\hline DM90 & LN871446 & A. radioresistens DSM6976 (X81666.1) & 460/465 (99) \\
\hline DM95 & LN871444 & P. stutzeri ATCC17588 (AF094748.1) & $1075 / 1082(99)$ \\
\hline \multicolumn{4}{|l|}{$r p o B$ gene $^{\text {b }}$} \\
\hline DD74 & KT456301 & P. stutzeri ATCC17588 (CP002881.1) & $1104 / 1111(99)$ \\
\hline DD75 & KT456289 & A. radioresistens DSM6976 (DQ207489.1) & $454 / 461(98)$ \\
\hline DD77 & KT456290 & A. calcoaceticus DSM30006 (DQ207474.1) & $460 / 460(100)$ \\
\hline DD78 & KT456291 & A. radioresistens DSM6976 (DQ207489.1) & $454 / 461(98)$ \\
\hline DD79 & KT456292 & A. radioresistens DSM6976 (DQ207489.1) & $408 / 415(98)$ \\
\hline DM80 & KT456293 & A. calcoaceticus DSM30006 (DQ207474.1) & $387 / 388(99)$ \\
\hline DM81 & KT456294 & A. calcoaceticus DSM30006 (DQ207474.1) & $424 / 432(98)$ \\
\hline DM82 & KT456295 & A. calcoaceticus DSM30006 (DQ207474.1) & $387 / 388(99)$ \\
\hline DM83 & KT456296 & A. calcoaceticus DSM30006 (DQ207474.1) & $385 / 389(99)$ \\
\hline DM84 & KT456297 & A. calcoaceticus DSM30006 (DQ207474.1) & $387 / 387(100)$ \\
\hline DM85 & KT456298 & A. calcoaceticus DSM30006 (DQ207474.1) & $459 / 460(99)$ \\
\hline DM86 & KT456299 & A. calcoaceticus DSM30006 (DQ207474.1) & $387 / 387(100)$ \\
\hline DM88 & KT456303 & P. stutzeri CCUG29243 (CP003677.1) & 1102/1111 (99) \\
\hline DM90 & KT456300 & A. radioresistens DSM6976 (DQ207489.1) & $454 / 461(98)$ \\
\hline DM95 & KT456302 & P. stutzeri $\mathrm{RCH} 2(\mathrm{CP} 003071.1)$ & $1053 / 1109(95)$ \\
\hline
\end{tabular}

${ }^{a}$ Gene sequences were deposited at the European Bioinformatics Institute (EBI) from EMBL-EBI, under the accession numbers indicated in brackets. ${ }^{b}$ Gene sequences were deposited at the National Centre for Biotechnology Information (NCBI) under the accession numbers indicated between brackets. 


\subsection{Identification of hydrocarbonoclastic isolates}

16S rRNA gene sequence analyses of bacterial isolates are summarized in Table 2. Strains DD74 and DM95 belong to genus Pseudomonas, showing 99\% identity to P. stutzeri ATCC 17588T. Isolate DM88 showed 99\% identity with $P$. chloritidismutans AW-1T. Strain DD76 showed $99.6 \%$ identity with Kocuria rosea DSM 20447T. Strains DD75, DD78, DD79 and DM90 exhibited $99 \%$ identity to $A$. radioresistens DSM 6976T. Isolate DM84 showed 99\% identity with $A c i-$ netobacter calcoaceticus ATCC 23055T.Strains DD77, DM80, DM81, DM82, DM83, DM85 and DM86 exhibited 97\% identity with Acinetobacter calcoaceticus

\section{ATCC 23055T}

For a more exhaustive identification of the isolates, analyses of housekeeping rpoB and rpoD gene sequences were performed. The $r p o B$ genes analyses of Acinetobacter isolates were in agreement with the 16S rRNA gene sequence analyses. Phylogenetic trees showed that the Acinetobacter strains belonged to two main evolutionary clusters, derived from $16 \mathrm{~S}$ rRNA (Figure $2 \mathrm{a}$ ) and $r p o B$ gene sequence analyses (Figure 2b). Strains DD77, DM80, DM81, DM82, DM83, DM84, DM85 and DM86 were clustered with A. calcoaceticus DSM 30006T. Strains DD75, DD78, DD79 and DM90 were clustered with $A$. $r a-$ dioresistens DSM 6976T.
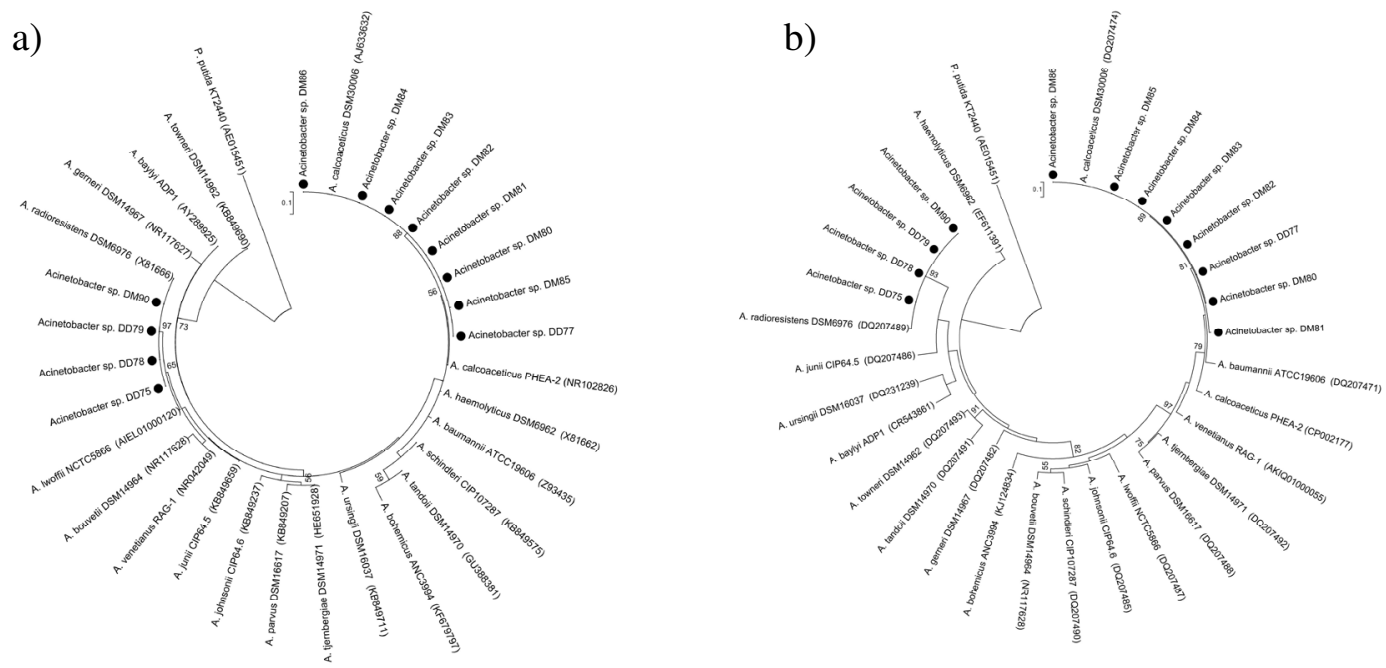

Figure 2. Identification of novel hydrocarbonoclastic Acinetobacter isolates by comparative sequence analyses. Phylogenetic trees based on v2-v3 regions of $16 \mathrm{~s}$ rRNA (a) and $\operatorname{rpo} B$ (b) gene sequences. Trees were constructed with ClustalW alignment and maximum-likelihood clustering. Bootstrap values $(1,000)$ are shown for each branch. 
Comparative rpoD, rpoB and 16S rRNA gene sequence analyses strongly suggested that Pseudomonas strains DD74 and DM95 are related to P. stutzeri. $16 \mathrm{~S}$ rRNA and rpoD gene analyses indicated that Pseudomonas sp. DM88 possessed high similarity to P. chloritidismutans AW-1T, whereas rpoB gene analyses showed that strain DM88 is related to P. stutzeri. Phylogenetic trees based on 16S rRNA gene (Figure 3a) and rpoB-rpoD concatenated genes (Figure 3b) sequences indicated that Pseudomonas strains DD74 and DM95 are clustered with P. stutzeri strains A1501 and RCH2, respectively. Although rpoB sequence analyses indicated that strain DM88 is closely related to the naphthalene-degrading type strain $P$. stutzeri CCUG29243T (Figure 3b), comparative 16S rRNA and rpoD analyses (Table 2) and phylogenetic trees based on $16 \mathrm{~S}$ rRNA and rpoB-rpoD-concatenated gene sequences strongly suggest that the isolate DM88 is highly related to P. chloritidismutans AW-1T (Figure 3). a)

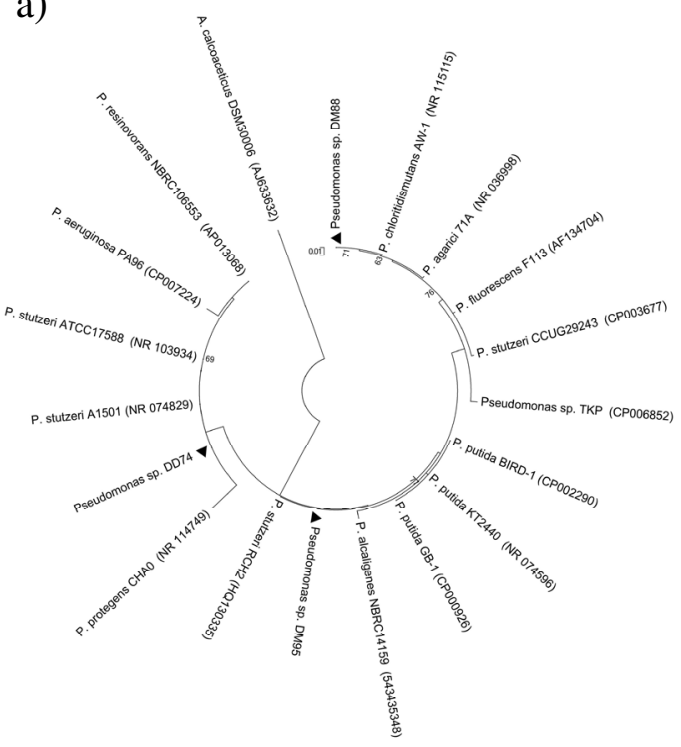

b)

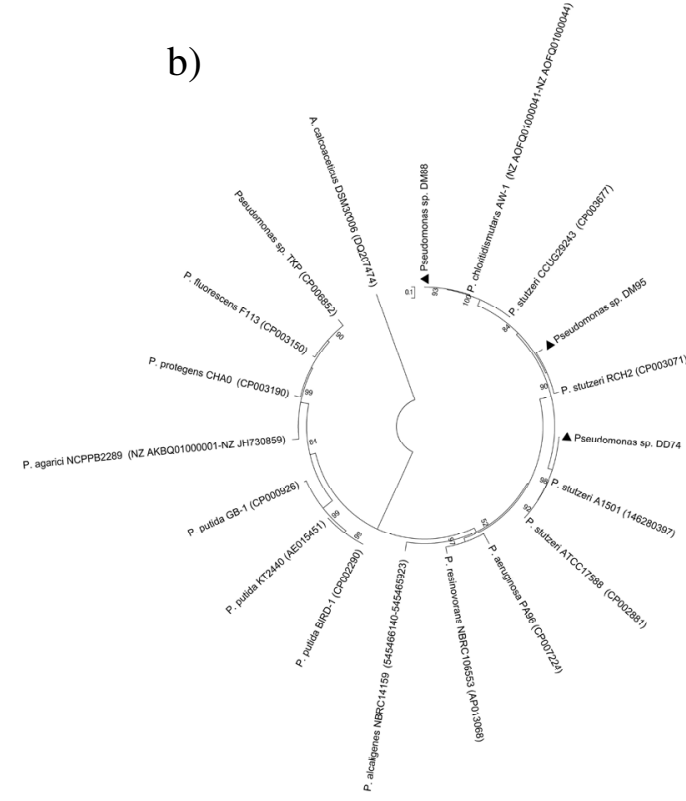

Figure 3. Identification of novel hydrocarbonoclastic Pseudomonas strains by comparative sequence analyses. Phylogenetic trees based on v2-v3 regions of 16s rRNA (a) and rpoB-rpoD-concatenated gene sequences. (b). Trees were constructed with ClustalW alignment and maximum-likelihood. Bootstrap values $(1,000)$ are shown for each branch. 


\subsection{Growth on hydrocarbons and heavy-metal tolerance}

The sixteen bacterial isolates showed growth in crude oil as sole carbon source. Most isolates were able to grow on an alkane or a polycyclic aromatic hydrocarbon (PAH). Eleven isolates showed growth on n-hexadecane as sole carbon and energy source (Table 3). $A$. radioresistens strains DD75 and DD79, Acinetobacter sp. strains DM81 and DM82 showed growth on nhexadecane and an aromatic hydrocarbon (i.e., fluorene or phenanthrene). P. stutzeri DD74 and Acinetobacter DM82 were able to grow on naphthalene and phenanthrene, respectively. A. radioresistens DD75 and DD79, Acinetobacter sp. DM81, P. chloritidismutans DM88 and $P$. stutzeri DM95 grew on fluorene.

Interestingly, thirteen isolates possessed tolerance to copper and eleven strains showed tolerance to cadmium. P. stutzeri DD74, K. rosea DD76, A. calcoaceticus
DM84, A. radioresistens strains DD78 and DM90, Acinetobacter sp. strains DD77, DM80, DM81, DM82, DM83 and DM85 possessed high tolerance to copper (MIC $1.6 \mathrm{mmol} \mathrm{l}^{-1}$ ) (Table 3). A. radioresistens DD75 and Acinetobacter sp. DM86 possessed moderate tolerance for copper (MIC 1.2 and $1.4 \mathrm{mmol} \mathrm{l}^{-1}$, respectively). In contrast, $A$. radioresistens DD79, P. stutzeri DM95 and P. chloritidismutans DM88 showed low tolerance to copper $\left(\mathrm{MIC}<0.80 \mathrm{mmol} \mathrm{l}^{-1}\right.$ ). A. radioresistens DD78, A. calcoaceticus DM84 and Acinetobacter sp. strains DD77, DM80, DM81, DM82 and DM85 exhibited a high tolerance to cadmium (MIC $1.3 \mathrm{mmol} \mathrm{1}^{-1}$ ), whereas P. stutzeri DD74, P. chloritidismutans DM88, A. radioresistens DD75 and K. rosea DD76 possessed moderate tolerance $\left(0.53 \mathrm{mmol} \mathrm{l}^{-1}\right)$ (Table 3). All strains were more sensitive than E. coli to $\mathrm{Hg}^{+2}\left(0.01 \mathrm{mmol} \mathrm{l}^{-1}\right), \mathrm{Ni}^{+2}\left(0.85 \mathrm{mmol} \mathrm{l}^{-1}\right)$ and $\mathrm{Pb}^{+2}$ $\left(0.53-2.4 \mathrm{mmol} \mathrm{l}^{-1}\right)$.

Table 3. Growth on hydrocarbons and heavy metal tolerance profile of bacterial isolates

\begin{tabular}{|c|c|c|c|c|c|c|c|c|c|c|c|c|c|c|c|c|c|}
\hline & \multicolumn{17}{|c|}{ Isolate } \\
\hline & 菕 & 㔯 & $\begin{array}{l}\stackrel{0}{0} \\
\text { مि }\end{array}$ & 命 & $\stackrel{\infty}{\hat{0}}$ & $\stackrel{\hat{\hat{O}}}{\hat{\hat{O}}}$ & $\sum_{0}^{\infty}$ & $\sum_{0}^{\infty}$ & $\sum_{\text {D. }}^{\infty}$ & $\sum_{0}^{\infty}$ & $\sum_{0}^{+}$ & $\sum_{0}^{\infty}$ & $\begin{array}{l}\sum_{0}^{\infty} \\
\sum_{0}^{\infty}\end{array}$ & $\begin{array}{l}\infty \\
\sum_{\text {D. }}^{\infty}\end{array}$ & $\sum_{0}^{\circ}$ & $\stackrel{n}{\sum_{0}}$ & $\underset{\tilde{\delta}}{\tilde{\delta}}$ \\
\hline \multicolumn{18}{|l|}{ Growth substrate } \\
\hline$n$-Octane & - & - & - & - & - & - & - & - & - & - & - & - & - & - & - & - & \\
\hline$n$-Hexadecane & - & + & - & + & + & + & - & + & + & + & + & + & + & - & + & - & \\
\hline Cyclohexane & - & - & - & - & - & - & - & - & - & - & - & - & - & - & - & - & \\
\hline Toluene & - & - & - & - & - & - & - & - & - & - & - & - & - & - & - & - & \\
\hline Naphthalene & + & - & - & - & - & - & - & - & - & - & - & - & - & - & - & - & \\
\hline Fluorene & - & + & - & - & - & + & - & + & - & - & - & - & - & + & - & + & \\
\hline Anthracene & - & - & - & - & - & - & - & - & - & - & - & - & - & - & - & - & \\
\hline Phenanthrene & - & - & - & - & - & - & - & - & + & - & - & - & - & - & - & - & \\
\hline Succinate & + & + & + & + & + & + & + & + & + & + & + & + & + & - & + & + & \\
\hline Glucose & - & + & + & + & + & + & + & + & + & + & + & + & + & + & + & + & \\
\hline \multicolumn{18}{|c|}{${ }^{b}$ Metal tolerance $(\mathrm{mM})^{\dagger}$} \\
\hline Copper $\left(\mathrm{CuCl}_{2}\right)$ & 1.6 & 1.2 & 1.6 & 1.6 & 1.6 & 0.8 & 1.6 & 1.6 & 1.6 & 1.6 & 1.6 & 1.6 & 1.4 & 0.53 & 1.6 & 0.8 & $1.0^{\ddagger}$ \\
\hline Nickel $\left(\mathrm{NiCl}_{2}\right)$ & 0.85 & 0.85 & 0.85 & 0.85 & 0.85 & 0.85 & 0.85 & 0.85 & 0.85 & 0.85 & 0.85 & 0.85 & 0.85 & 0.85 & 0.85 & 0.85 & $1.0^{\ddagger}$ \\
\hline Cadmium $\left(\mathrm{CdCl}_{2}\right)$ & 0.53 & 0.53 & 0.53 & 1.3 & 1.3 & 0.22 & 1.3 & 1.3 & 1.3 & 0.3 & 1.3 & 1.3 & 0.3 & 0.53 & 0.30 & 0.22 & $0.50^{\ddagger}$ \\
\hline Lead $\left(\mathrm{Pb}\left(\mathrm{NO}_{3}\right)_{2}\right)$ & 0.50 & 0.50 & 0.50 & 2.4 & 0.50 & 1.45 & 0.50 & 1.0 & 0.50 & 0.50 & 0.50 & 1.0 & 0.50 & 0.50 & 0.50 & 1.45 & $5.0^{\ddagger}$ \\
\hline Mercury $\left(\mathrm{HgCl}_{2}\right)$ & 0.01 & 0.01 & 0.01 & 0.01 & 0.01 & 0.01 & 0.01 & 0.01 & 0.01 & 0.01 & 0.01 & 0.01 & 0.01 & 0.01 & 0.01 & 0.01 & $0.01^{*}$ \\
\hline
\end{tabular}

${ }^{*}+$, Growth; -, No growth. $†$ Minimal inhibitory concentration (MIC) on the corresponding metal ion. $\ddagger$ Reference MIC value in $E$. coli (Nies, 1999). 


\section{Discussion}

Hydrocarbon-degrading bacteria possessing heavy metal tolerance are essential for bioremediation of petroleum-contaminated soils that contain high levels of heavy metals. In this study, sixteen hydrocarbonoclastic strains were isolated by enrichment using crude oil from a decantation sludge and a diesel motor oil as sole carbon sources. All bacterial isolates showed resistance to antibiotics (2-5) tested. This is not surprising, since antibiotic resistance is widely spread in environmental bacteria. The extensive use of antibiotics in agriculture in the Aconcagua valley is an important source of antibiotics in the environment.

Based on 16S rRNA, $r p o B$ and $r p o D$ gene sequences analyses, bacterial isolates were affiliated to the genera Pseudomonas and Acinetobacter. Isolates from the genus Acinetobacter were the most abundant (Table 2). In a previous study, one diesel-enrichment from a hydrocarbons-polluted soil at Aconcagua river estuary was mainly composed by Pseudomonadaceae, whereas a second diesel-enrichment was composed by Acinetobacter, Rhizobiaceae and Moraxellaceae, suggesting that Pseudomonas and Acinetobacter are the predominant cultivable hydrocarbonoclastic bacteria in these soils (Fuentes et al., 2016). 16S rRNA and housekeeping gene sequences analyses indicated that isolated strains were affiliated to the genera $\mathrm{Aci}$ netobacter, Pseudomonas and Kocuria. Three isolates (DD74, DM88 and DM95) were affiliated to the genus Pseudomonas according to $16 \mathrm{~S}$ rRNA sequence analysis. These results were in agreement with the 16S rRNA and rpoB phylogenetic analyses, indicating that DD74 and DM95 strains are closely related to the type strains $P$. stutzeri A1501 and $\mathrm{RCH} 2$, respectively (Figure 3). The genome of root-associated P. stutzeri strain A1501 contains genes involved in the degradation of aromatic compounds (Yan et al., 2008). P. stutzeri $\mathrm{RCH} 2$ was isolated from a retired nuclear re- actor complex with high levels of heavy metals contamination, particularly chromium (Han et al., 2010). Although morphological differences were observed between DD74 and DM95 strains, comparative sequence analyses support that these isolates are highly related to P. stutzeri reference strains. P. stutzeri typically form irregular, hard and dry colonies. However, the colonies shape can change after repeated transfers in laboratory conditions, becoming smooth and pale in color, phenomenon that has been described as colonial dissociation (Lalucat et al., 2006).

According to $r p o B$ gene sequence analysis, strain DM88 is closely related (99\%) to the type strain $P$. stutzeri CCUG29243 (Table 2). However, comparative $16 \mathrm{~S}$ rRNA sequences and rpoB-rpoD-concatenated tree indicate that DM88 strain is highly related to the type strain P. chloritidismutans AW-1 (Table 2 and Figure 3). Wolterink et al. (2002) reported the chlorate reducer $P$. chloritidismutans sp. nov. AW-1T, possessing $100 \% 16 \mathrm{~S}$ rRNA gene sequence identity to P. stutzeri DSM 50227 and $98.6 \%$ to P. stutzeri DSM 5190T. However, a DNA-DNA hybridization approach along with physiological and biochemical analyses indicated that $P$. chloritidismutans AW$1 \mathrm{~T}$ and P. stutzeri strains belong to different species (Wolterink et al., 2002).

In this study, phylogenetic analysis using $16 \mathrm{~S}$ rRNA and $r p o B$ sequences indicated that most of the $A c i$ netobacter isolates were related to the type strain $A$. calcoaceticus DSM30006T and the phenol-degrader A. calcoaceticus PHEA-2, which were isolated by quinate enrichment from soil and wastewater (Zhan et al., 2011), respectively (Figure 2). Four isolates were closely related with $A$. radioresistens DSM 6976T, which was isolated from cotton sterilized by $\gamma$-radiation (Chan et al., 2012) and grew slightly on diesel (Mara et al., 2012). However, a 97\% identity in $16 \mathrm{~S}$ rRNA gene sequences analyses suggested that seven isolates (DD77, DM80, DM81, DM82, DM83, 
DM85 and DM86) may probably represent novel species. A previous report suggested that a $16 \mathrm{~S}$ rRNA genes similarity of $<97 \%$ likely represents a novel species (Drancourt et al., 2004). Genotypic characterization based on PCR amplification and sequence analyses of 16S rRNA gene has been a method widely used for the phylogenetic relationships and identifications of bacteria. However, rRNA gene sequence analyses do not offer resolution for definitive species level identification. Different methodologies for phenotypic characterizations, such as cell morphology and metabolic profiling, allow differentiation of taxa at higher level (Moore et al., 2010). Nevertheless, further analysis will be needed for the identification of these Acinetobacter isolates.

Differences in antibiotic resistance and heavy-metal tolerance profiles were found among these Acinetobacter isolates. For example, DM81 and DM82 isolates possessed different heavy metal tolerance profile (Table 3). Interestingly, the isolates DM81 and DM82 were able to grow on fluorene and phenanthrene, respectively (Table 3). Strains from genera Acinetobacter and Pseudomonas showed also interesting catabolic abilities along with heavy-metal tolerance. In this study, P. stutzeri sp. strains DD74 and DM95 were able to grow on naphthalene and fluorene, respectively (Table 2). P. stutzeri AN10 and P. putida G7 have been model strains for the study of naphthalene degradation (Lalucat et al., 2006). However, $P$. stutzeri able to metabolize fluorene has been poorly described (Stringfellow and Aitken, 1995). Interestingly, $P$. chloritidismutans strain DM88 was able to grow on fluorene (Table 2). To our knowledge, this is the first study that describes a $P$. chloritidismutans strain able to grow on fluorene. Previous reports have described the isolation of PAH-degrading bacteria possessing heavy metal tolerance from co-contaminated sites. Naphthalene-degrading and mercury-tolerant Clavibacter, Arthrobacter and Acidocella strains were isolated from a contaminated coal storage pile site (Dore et al., 2003). Paenibacillus and Pseudomonas strains isolated from a PAH-polluted lagoon were able to degrade naphthalene and possessed tolerance to lead (Pepi et al., 2009). Although strains from the Pseudomonas genus that aerobically degrade diverse aromatic hydrocarbons have been reported, in this study we describe two strains (P. stutzeri DD74 and P. chloritidismutans DM88) that are able to metabolize fluorene and possess tolerance to copper and cadmium. Interestingly, P. stutzeri RCH2 is tolerant to copper and zinc (Vaccaro et al., 2016).

Although $\mathrm{n}$-hexadecane degradation by Acinetobacter strains has been widely reported (Koma et al., 2001; Kang and Park, 2010), reports of Acinetobacter strains possessing heavy metal tolerance together with PAH degradation abilities are scarce. Acinetobacter strains tolerant to chromium, nickel and cadmium that are useful for heavy metal bioremediation have been described (Bhattacharya and Gupta, 2013). In this study, almost all Acinetobacter isolates were able to grow on n-hexadecane as sole carbon source and possessed tolerance to copper and cadmium. Remarkably, Acinetobacter sp. strain DM81 grew on n-hexadecane and fluorene, whereas Acinetobacter sp. strain DM82 showed growth on $\mathrm{n}$-hexadecane and phenanthrene as sole carbon sources, both possessing tolerance to copper and cadmium. A. radioresistens DD75 showed growth on n-hexadecane, fluorene and tolerance to copper and cadmium (Table 3). High levels of copper in crude oil-polluted soils from Aconcagua river estuary are probably related to copper mining activities along the Aconcagua Valley (Fuentes et al., 2015). Copper, calcium and arsenic are main drivers of microbial communities in Aconcagua river estuary soils (Fuentes et al., 2015). Accordingly, Sphingomonas, Stenotrophomonas and Arthrobacter strains possessing high copper resistance (MIC 3.1 - $4.7 \mathrm{mmol}$ $\mathrm{L}^{-1}$ ) have been isolated from copper-polluted soils at 
Aconcagua valley (Altimira et al., 2012). Therefore, it was not surprising to find that most of the hydrocarbonoclastic isolates the Aconcagua river mouth possessed heavy-metal tolerance. The strains isolated and characterized in this study are promising biocatalysts for bioremediation.

\section{Conclusions}

In this study, sixteen hydrocarbonoclastic bacteria belonging mainly to Acinetobacter and Pseudomonas genera has been isolated from a crude oil-polluted soil close to Aconcagua river mouth, Central Chile. Isolated strains were able to grow on different petroleum hydrocarbon components (i.e., n-hexadecane or a PAH). Notably, most bacterial isolates possessed tolerance to copper and cadmium, which represents an advantage for the design of bioremediation strategies. Future studies will include bioaumentation using these novel Acinetobacter and Pseudomonas strains for the clean-up of petroleumpolluted sites that contain heavy metals.

\section{Acknowledgements}

The authors thank Pola Miralles for support in sampling. This study was financially supported by FONDECYT 1151174 \& 1110992, CN\&BS and USM 131562, 31342 \& 131109. V. Méndez, S. Fuentes, V. Morgante and M. Hernández acknowledge financial support from CONICYT, MECESUP and RIABIN fellowships.

\section{References}

Altimira, F., Yáñez, C., Bravo, G., González, M., Rojas, L.A., Seeger, M. 2012. Characterization of copper-resistant bacteria and bacterial commu-
Amezcua-Allieri, M.A., Lead, J.R., RodríguezVázquez, R. 2005. Impact of microbial activity on copper, lead and nickel mobilization during the bioremediation of soil PAHs. Chemosphere 61, 484-491.

Atlas, R., Philp, J. 2005. Bioremediation: applied microbial solutions for real-world environmental cleanup. ASM Press, Washington D.C, USA. 399 p.

Bhattacharya, A., Gupta, A. 2013. Evaluation of Acinetobacter sp. B9 for $\mathrm{cr}$ (vi) resistance and detoxification with potential application in bioremediation of heavy-metals-rich industrial wastewater. Environ. Sci. Pollut. Res. 20, 6628-6637.

Chan, J.Z., Halachev, M.R., Loman, N.J., Constantinidou, C., Pallen, M.J. 2012. Defining bacterial species in the genomic era: insights from the genus Acinetobacter. BMC Microbiol. 12, 302.

Dore, S.Y., Clancy, Q.E., Rylee, S.M., Kulpa, C.F. 2003. Naphthalene-utilizing and mercury-resistant bacteria isolated from an acidic environment. Appl. Microbiol. Biotechnol. 63, 194-199.

Drancourt, M., Berger, P., Raoult, D. 2004. Systematic 16s rRNA gene sequencing of atypical clinical isolates identified 27 new bacterial species associated with humans. J. Clin. Microbiol. 42, 2197-2202.

Fuentes, S., Méndez, V., Aguila, P., Seeger, M. 2014. Bioremediation of petroleum hydrocarbons: catabolic genes, microbial communities, and applications. Appl. Microbiol. Biotechnol. 98, 47814794.

Fuentes, S., Ding, G.C., Cárdenas, F., Smalla, K., Seeger, M. 2015. Assessing environmental drivers of microbial communities in estuarine soils of the Aconcagua river in Central Chile. FEMS Microbiol. Ecol. 91, 1-12.

Fuentes, S., Barra, B., Caporaso, J.G., Seeger, M. 2016. From rare to dominant: a fine-tuned soil bacterial bloom during petroleum hydrocarbon 
bioremediation. Appl. Environ. Microbiol. 82, 888-896.

Han, R., Geller, J.T., Yang, L., Brodie, E.L., Chakraborty, R., Larsen, J.T., Beller, H.R. 2010. Physiological and transcriptional studies of $\mathrm{cr}(\mathrm{vi})$ reduction under aerobic and denitrifying conditions by an aquifer-derived Pseudomonad. Environ. Sci. Technol. 44, 7491-7497.

Hernández, M., Jia, Z., Conrad, R., Seeger, M. 2011. Simazine application inhibits nitrification and changes the ammonia-oxidizing bacterial communities in a fertilized agricultural soil. Fems Microbiol. Ecol. 78, 511-519.

Kang, Y.S., Park, W. 2010. Contribution of quorumsensing system to hexadecane degradation and biofilm formation in Acinetobacter sp. strain DR1. J. Appl. Microbiol. 109, 1650-1659.

Koma, D., Hasumi, F., Yamamoto, E., Ohta, T., Chung, S.Y, Kubo, M. 2001. Biodegradation of long-chain n-paraffins from waste oil of car engine by Acinetobacter sp. J. Biosci. Bioeng. 91, 94-96.

Lalucat, J., Bennasar, A., Bosch, R., García-Valdés, E., Palleroni, N.J. 2006. Biology of Pseudomonas stutzeri. Microbiol. Mol. Biol. Rev. 70, 510-547.

Mara, K., Decorosi, F., Viti, C., Giovannetti, L., Papaleo, M.C., Maida, I., Perrin, E., Fondi, M., Vaneechoutte, M., Nemec, A., Van den Barselaar, M., Dijkshoorn, L., Fani, R. 2012. Molecular and phenotypic characterization of Acinetobacter strains able to degrade diesel fuel. Res. Microbiol. 163, 161-172.

Moore, E.R., Mihaylova, S.A., Vandamme, P., Krichevsky, M.I., Dijkshoorn, L. 2010. Microbial systematics and taxonomy: relevance for a microbial commons. Res. Microbiol. 161, 430-438.

Morgante, V., López-López, A., Flores, C., González, M., González, B., Vásquez, M., Rosselló-Mora, R., Seeger, M. 2010. Bioaugmentation with Pseudomonas sp. strain MHP41 promotes simazine attenuation and bacterial community changes in agricultural soils. Fems Microbiol. Ecol. 71, 114-126.

Nies, D.H. 1999. Microbial heavy-metal resistance. Appl. Microbiol. Biotechnol. 51, 730-750.

Olaniran, A.O., Balgobind, A., Pillay, B. 2013. Bioavailability of heavy metals in soil: impact on microbial biodegradation of organic compounds and possible improvement strategies. Int. J. Mol. Sci. 14, 10197-10228.

Palma-Fleming, H., Cornejo, C., González, M., Gutierrez, E., Sericano, J.L., Seeger, M. 2008. Polyciclic aromatic hydrocarbons and polychlorinated biphenyls in coastal environments of Valdivia and Valparaíso, Chile. J. Chil. Chem. Soc. 53, 1533-1538.

Pepi, M., Lobianco, A., Renzi, M., Perra, G., Bernardini, E., Marvasi, M., Gasperini, S., Volterrani, M., Franchi, E., Heipieper, H.J., Focardi, S.E. 2009. Two naphthalene degrading bacteria belonging to the genera Paenibacillus and Pseudomonas isolated from a highly polluted lagoon perform different sensitivities to the organic and heavy metal contaminants. Extremophiles. 13, 839-848.

Ponce, B.L., Latorre, V.K., González, M., Seeger, M. 2011. Antioxidant compounds improved pcbdegradation by Burkholderia xenovorans strain LB400. Enzym. Microb. Technol. 49, 509-516.

Ratajczak, A., Geißdörfer, W., Hillen, W. 1998. Alkane hydroxylase from Acinetobacter sp. strain ADP1 is encoded by alkM and belongs to a new family of bacterial integral-membrane hydrocarbon hydroxylases. Appl. Environ. Microbiol. 64, 1175-1179.

Rojas, L.A., Yáñez, C., González, M., Lobos, S., Smalla, K., Seeger, M. 2011. Characterization of the metabolically modified heavy metal-resistant Cupriavidus metallidurans strain MSR33 generated for mercury bioremediation. PLoS ONE 6, e17555. doi: 10.1371/journal.pone.0017555. 
Seeger, M., Hernández, M., Méndez, V., Ponce, B., Córdova, M., González, M. 2010. Bacterial degradation and bioremediation of chlorinated herbicides and biphenyls. J. Soil Sci. Plant Nutr. 10, 320-332.

Silva, I.S., Santos, E.C. Menezes, C.R., de Faria, A.F., Franciscon, E., Grossman, M., Durrant, L.R. 2009. Bioremediation of a polyaromatic hydrocarbon contaminated soil by native soil microbiota and bioaugmentation with isolated microbial consortia. Bioresour. Technol. 100, 4669-4675.

Singleton, D.R., Ramirez, L.G., Aitken, M.D. 2009. Characterization of a polycyclic aromatic hydrocarbon degradation gene cluster in a phenanthrene-degrading Acidovorax strain. Appl. Environ. Microbiol. 75, 2613-2620..

Stringfellow, W.T., Aitken, M.D. 1995. Competitive metabolism of naphthalene, methylnaphthalenes, and fluorene by phenanthrene-degrading pseudomonads. Appl. Environ. Microbiol. 61, 357-362.

Vaccaro, B.J., Lancaster, W.A., Thorgersen, M.P., Zane, G.M., Younkin, A.D., Kazakov, A.E., Wetmore, K.M., Deutschbauer, A., Arkin, A.P., Novichkov, P.S., Wall, J.D., Adams M.W.W. 2016. Novel metal mation resistance systems from mutant fitness analysis of denitrifying Pseudomonas stutzeri. Appl. Environ. Microbiol. 82, 6046-6056. van Beilen, J.B., Panke, S., Lucchini, S., Franchini, A.G., Röthlisberger, M., Witholt, B. 2001. Analysis of Pseudomonas putida alkane-degradation gene clusters and flanking insertion sequences: evolution and regulation of the alk genes. Microbiology. 147, 1621-1630.

Velázquez, F., De Lorenzo, V., Valls, M. 2006. The mxylene biodegradation capacity of Pseudomonas putida $\mathrm{mt}-2$ is submitted to adaptation to abiotic stresses: evidence from expression profiling of xyl genes. Environ. Microbiol. 8, 591-602.

Wolterink, A.F.W.M., Jonker, A.B., Kengen, S.W.M., Stams, A.J.M. 2002. Pseudomonas chloritidismutans sp. nov., a non-denitrifying, chloratereducing bacterium. Int. J. Syst. Evol. Microbiol. 52, 2183-2190.

Yan, Y., Yang, J., Dou, Y., Chen, M., Ping, S., Peng, J., Lu, W., Zhang, W., Yao, Z., Li, H., Liu, W., He, S., Geng, L., Zhang, X., Yang, F., Yu, H., Zhan, Y., Li, D., Lin, Z., Wang, Y., Elmerich, C., Lin, M., Jin, Q. 2008. Nitrogen fixation island and rhizosphere competence traits in the genome of rootassociated Pseudomonas stutzeri a1501. Proc. Natl. Acad. Sci. U.S.A. 105, 7564-7569.

Zhan, Y., Yan, Y., Zhang, W., Yu, H., Chen, M., Lu, W., Ping, S., Peng, Z., Yuan, M., Zhou, Z., Elmerich, C., Lin, M. 2011. Genome sequence of Acinetobacter calcoaceticus phea-2, isolated from industry wastewater. J. Bacteriol. 193, 2672-2673. 